\title{
MÔ HÌNH HÓA Bộ TRAO ĐỔI NHIỆT ĐÚNG KIỂU VỎ VÀ ỐNG
}

\author{
LÊ TUẤN PHƯƠNG NAM \\ Khoa Công nghệ Co khí, Trường Đại học Công nghiệp thành phố Hồ Chí Minh; \\ letuanphuongnam@iuh.edu.vn
}

Tóm tắt. Bộ trao đổi nhiệt (BTĐN) kiểu vỏ và ống đã được sử dụng phổ biến trong ngành công nghiệp. Trong quá trình ứng dụng thiết bị này vào thực tiễn người vận hành phải biết xử lý "động" cho bộ trao đồi nhiệt nhằm dự đoán được khả năng đáp ứng khả năng làm việc khi có sự thay đổi về lưu lượng, nhiệt độ, áp suất, vv... của dòng lưu chất đi qua BTĐN. Mô hình hóa và mô phỏng các xử lý động của một bộ trao đổi nhiệt kiểu vỏ và ông nằm ngang đã được một số tác giả công bô. Trong bài báo này, tác giả trình bày một mô hình động của bộ trao đổi nhiệt kiểu vỏ và ống thẳng đứng, lưu chất nóng là hơi nước và lưu chất lạnh là nước ngưng. Mô hình được trình bày trong bài báo nhằm dự đoán áp suất hơi nước và mức nước ngưng trong bộ trao đổi nhiệt. Các kết quả mô phỏng thì tương đồng với dữ liệu thực nghiệm.

Từ khóa. Bộ trao đổi nhiệt kiểu vỏ và ống, kiểm soát thể tích, mô hình toán học, áp suất hơi nước, chiều cao nước ngưng.

\section{MODELING OF A VERTICAL SHELL-AND-TUBE HEAT EXCHANGER}

\begin{abstract}
The shell and tube heat exchanger has been commonly used in the industry. It is important to know the dynamic behavior of the heat exchanger in order to predict how the system will respond to a change in flow rate, temperature, pressure, etc.... Modeling and simulation of the dynamic behavior of a horizontal shell-and-tube heat exchanger are presented in the literature. In this paper, a dynamic modeling of a vertical shell-and-tube heat exchanger is presented. The hot fluid is steam and the cold fluid is water. The objective of this model is to predict steam pressure and level of the condensate water in the heat exchanger. The simulation results of steam pressure and level of condensate water agree well with those of experimental data.
\end{abstract}

Keywords. Shell and tube heat exchanger, control volume, modeling, steam pressure, height of condensate water.

\section{GIỚI THIỆU}

Các bộ trao đổi nhiệt kiểu vỏ và ống rất thường được sử dụng trong ngành công nghiệp. Mô hình hóa và mô phỏng các ứng xử động của bộ trao đổi nhiệt vỏ và ống đã được trình bày trong các công bố [1-10] nhưng chỉ cho các bộ trao đổi nhiệt kiểu ống và vỏ nằm ngang và có vách ngăn. Các mô hình này đều dựa trên nguyên lý bào toàn khối lượng và năng lượng. Trong [1], bộ trao đổi nhiệt kiểu vỏ và ống nằm ngang với giả định rằng các lưu chất là lưu chất một pha không nén được. Mô hình này có đủ linh hoạt để cho phép so sánh cả định tính và định lượng của các thiết kế khác nhau cho các mục tiêu thiết kế khác nhau. Tuy nhiên có một hạn chế trong mô hình, nó không thể dự đoán lưu lượng dòng chảy của lưu chất lỏng. Một mô hình đã được phát triển trong [2] để khắc phục vấn đề này. Trong nghiên cứu [2] bộ trao đổi nhiệt kiểu ống nằm ngang có vách ngăn dùng hơi nước như lưu chất nóng và nước như lưu chất lạnh. Mô hình trong [2] có thể dự đoán lưu lượng dòng chảy, hơi nước và áp suât hơi nước. Mô hình hóa bộ trao đổi nhiệt dựa trên các nguyên lý bảo toàn cho khối lượng và năng lượng cho mỗi thể tích kiểm soát (Control Volume - $\mathrm{CV}$ ) được trình bày trong tài liệu [1-5]. Một cách khác để có thể mô hình hóa và mô phỏng bộ trao đổi nhiệt là dùng phương pháp tính toán động lực học lưu chất (Computational Fluid Dynamics $\mathrm{CFD}$ ) được trình bày trong tài liệu [6-10]. Trong cách $\mathrm{CFD}$, bộ trao đổi nhiệt ngang kiểu ống và vỏ được chia lưới thay vì là các thể tích kiểm soát và quá trình mô hình hóa được xây dựng trên các phương trình bảo toàn khối lượng, động lượng và năng lượng. Các mô phỏng CFD này sẽ dự báo được các trường nhiệt độ và vận tốc của lưu chất trong bộ trao đổi nhiệt. Trong bài báo này, một mô hình toán học cho một bộ trao đổi nhiệt kiểu vỏ và ống thẳng đứng và không có vách ngăn được trình bày. Bộ trao đổi nhiệt cũng 
được chia thành các "thể tích kiểm soát" và các phương trình bảo toàn khối lượng và năng lượng được áp dụng cho mỗi thể tích kiểm soát. Lưu chất nóng là hơi nước và lưu chất lạnh là nước ngưng. Chúng tôi không mô phỏng tốc độ của lưu chất hoặc các lực tác động lên trên thành vỏ và ống nên nguyên lý bảo toàn động lượng không được xem xét trong mô hình toán này. Mục tiêu của mô hình này là để dự đoán lưu lượng hơi nước và nước ngưng, áp suất hơi nước và nhiệt độ nước ngưng. Hơn nữa, mức nước ngưng tụ trong bộ trao đổi nhiệt vỏ và ống thẳng đứng cũng được mô phỏng. Tuy nhiên vì hạn chế của dữ liệu thực nghiệm, chúng tôi chỉ trình bày các kết quả mô phỏng của áp suất hơi nước và chiều cao của mức nước ngưng trong bộ trao đổi nhiệt mà được so sánh với kết quả thực nghiệm trong [11].

Bộ trao đổi nhiệt đứng kiểu vỏ và ống là một thành phần chính của hệ thống trao đổi nhiệt trong hình 1 được xây dựng tại trung tâm nhiệt của Trường Công Nghệ Cao (Ecole de Technologie Superieure ETS), Montreal, Canada [12] với mục đích cân chỉnh "calibrate" lưu lượng kế hơi nước dựa trên đo trọng lượng của nước ngưng $[13,14]$. Hệ thống này bao gồm một mạch dòng hơi nước và một mạch nước làm mát nối với nhau bằng một bộ trao đổi nhiệt đứng kiểu vỏ và ống (xem hình 1 ). Các thành phần chính và hoạt động của hệ thống như sau:

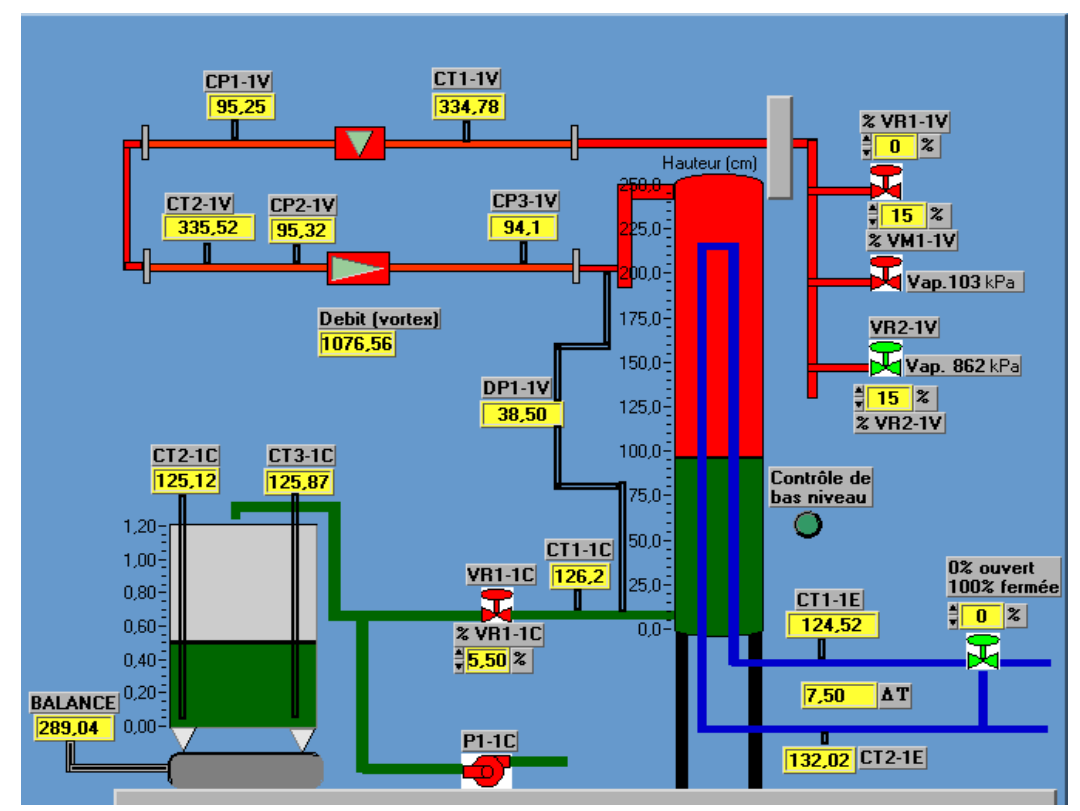

Hình 1. Sơ đồ hệ thống đo lưu lượng hơi nước bằng phương pháp trọng lượng [12].

Hơi nước được đưa vào hệ thống bằng cách mở một trong ba van được hiển thị bởi VR1-1V, VM1$1 \mathrm{~V}$ và $\mathrm{VR2}-1 \mathrm{~V}$ trong hình 1 . Trong nghiên cứu ở bài báo này van VR2-1V với áp suất không đổi $\mathrm{p}_{0}=$ 862Pa. Hơi nước này ở trạng thái bão hòa, áp suất (hoặc nhiệt độ) được điều khiển bởi vị trí mở của van. Hơi nước đi qua lưu lượng kế được biểu thị bằng biểu tượng $\square$ trước khi đi vào phía trên của bộ trao đổi nhiệt đứng kiểu ống và vỏ. Nhiệt độ và áp suất hơi nước trước và sau khi đi qua lưu lượng kế đều được đo và hiển thị bởi $\mathrm{CT} 1-1 \mathrm{~V}, \mathrm{CP} 1-1 \mathrm{~V}, \mathrm{CT} 2-1 \mathrm{~V}, \mathrm{CP} 2-1 \mathrm{~V}$ và $\mathrm{CP} 3-1 \mathrm{~V}$ thể hiện trên hình 1 . Bằng cách truyền nhiệt cho nước làm mát, hơi nước ngưng tụ ở phía dưới cùng của bộ trao đổi nhiệt và được thải ra từ ống thoát của phía vỏ bộ trao đổi nhiệt và chảy vào một bể chứa (xem phần dưới cùng bên trái của hình 1$)$. Nước làm mát chảy vào đầu dưới của bộ trao đổi nhiệt từ ống dẫn vào bằng cách mở van lắp trên đó mà chỉ số $0 \%$ là mở (Ouvert) trên hình 1 . Bên trong bộ trao đổi nhiệt, nước làm mát phân ra và chảy thành 24 ống nhỏ (không được thể hiện chi tiết), sau đó xuống đến 24 ống khác, nối lại với nhau và rời khỏi bộ trao đổi nhiệt bằng đường ống thoát. Nhiệt độ của nước làm mát trước khi vào và ra khỏi bộ trao đổi nhiệt được đo và hiện thị ở nhiệt kế $\mathrm{CT} 1-1 \mathrm{E}$ và $\mathrm{CT} 2-1 \mathrm{E}$. Độ chênh lệch áp suất giữa hơi nước và nước ngưng được hiện thị qua DP1-1V trên hình 1. Một cái cân (Balance trên hình 1) được sử dụng để đo trọng lượng của nước ngưng thu được theo thời gian để hiệu chỉnh "calibrate" lưu lượng nước ngưng. Chiều cao của nước ngưng trong bộ trao đổi nhiệt đứng được chỉ báo bằng một áp kế (một ống trong suốt thẳng đứng hiển thị bên trái của bộ trao đổi nhiệt) và được điều khiển bởi vị trí mở của van VR1-1C, được lắp đặt trên ống thoát nước ngưng. Khi chiều cao này trở nên ổn định, trọng lượng ngưng tụ được đo bằng cân bằng 
cũng chỉ ra tốc độ lưu lượng hơi nước. Nhiệt độ của nước ngưng đi ra khỏi bộ trao đổi nhiệt và trong thùng chứa đều được đo và hiện thị trên CT1-1C, CT3-1C và CT2-1C. Một hệ thống thu thập dữ liệu tích hợp cho phép ghi lại các giá trị số của các thông số hoạt động đã chọn như áp suất, nhiệt độ, mức nước ngưng tụ ...

\section{MÔ HİNH TOÁN HỌC CỦA Bộ TRAO ĐỔI NHIỆT ĐỨNG KIỂU VỎ VÀ ỐNG}

Bộ trao đổi nhiệt cũng được chia thành các "thể tích kiểm soát" khác nhau, nhưng trong bộ trao đổi ngang, mỗi thể tích này gồm có cả hai pha lỏng và hơi. Trong bộ trao đổi thẳng đứng, chúng tôi có thể chia một số thể tích kiểm soát cho pha hơi nước và một số thể tích kiểm soát cho pha nước ngưng. Hình 2 trình bày cách chia các thể tích kiểm soát của bộ trao đổi kiểu vỏ và ống thẳng đứng. Chúng ta có thể phân chia pha nước ngưng vào $\mathrm{n}$ thể tích kiểm soát bằng nhau và pha hơi nước vào $\mathrm{m}$ thể tích kiểm soát. Các thông số chính của bộ trao đổi nhiệt thẳng đứng kiểu vỏ và ống là đường kính vỏ $\mathrm{D}_{\mathrm{s}}=0.3048 \mathrm{~m}$, đường kính ống $\mathrm{D}_{\mathrm{t}}=0.01905 \mathrm{~m}$, chiều cao của bộ trao đổi nhiệt $\mathrm{L}=2.5 \mathrm{~m}$ và số ống trong bộ trao đổi nhiệt $\mathrm{N}=48$.

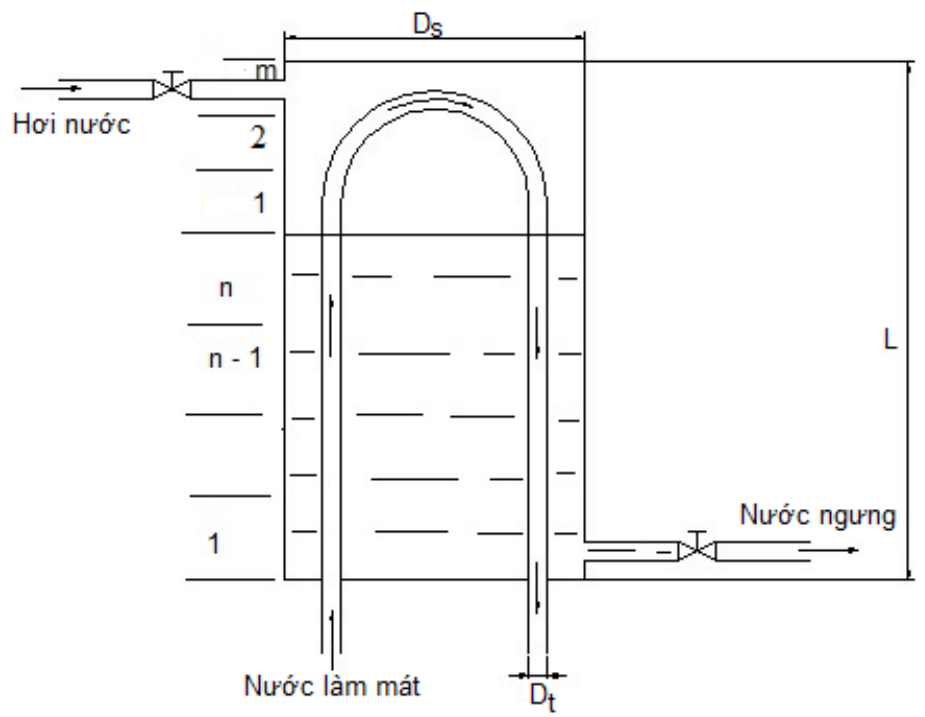

Hình 2. Sơ đồ chia thể tích kiểm soát của bộ trao đổi nhiệt đứng kiểu ống và vỏ.

Bộ trao đổi nhiệt được chia nhỏ thành các thể tích kiểm soát $(\mathrm{C} . \mathrm{V})$ và các phương trình được phát triển cho các đại lượng vật lý tại các biên của các C.V này. Một cách tổng quát, việc tăng số lượng C.V cho kết quả tốt hơn nhưng cần nhiều phương trình được phát triển và tăng thêm thời gian tính toán. Trong mô hình toán ở đây chúng tôi chọn $n=2$ và $m=1$, vì vậy có 3 thể tích kiểm soát $(C . V)$ cho mặt vỏ. Thể tích chứa nước ngưng được chia thành hai thể tích điều khiển bằng nhau $(\mathrm{C} . \mathrm{V} 1$ và $\mathrm{C} . \mathrm{V} 2)$ và hơi nước ở trên nước ngưng được lấy làm một thể tích kiểm soát (C.V3 trong hình 3 ). Tương ứng với $3 \mathrm{C}$.V cho vỏ chúng ta có $5 \mathrm{C}$.V cho ống như trên hình 3.

Với tổng cộng 8 thể tích kiểm soát được chia trên cho vỏ và ống như trên hình 3 chúng ta có tổng cộng 22 ẩn số như sau:

$\mathrm{T}_{\mathrm{c} 1}, \mathrm{~T}_{\mathrm{c} 2}$ - Nhiệt độ trung bình của nước ngưng trong C.V1 và C.V2

$\mathrm{P}_{\mathrm{s}}, \mathrm{T}_{\mathrm{s}}$ - Áp suất và nhiệt độ hơi nước bảo hòa trong C.V3.

$\mathrm{T}_{0}, \mathrm{~T}_{1}, \mathrm{~T}_{2}, \mathrm{~T}_{3}, \mathrm{~T}_{4}, \mathrm{~T}_{5}$ - Nhiệt độ của nước làm mát trong ống ở các biên của mỗi C.V.

$\mathrm{m}_{\mathrm{s}}$ - lưu lượng vào của hơi nước, dấu chấm “•” thể hiện đạo hàm theo thời gian dm/dt.

$\dot{\mathrm{m}}_{\mathrm{c} 3}, \dot{\mathrm{m}}_{\mathrm{c} 2}$ - lưu lượng nước ngưng ở biên $\mathrm{C} . \mathrm{V} 3$ với $\mathrm{C} . \mathrm{V} 2$ và lưu lượng ở biên $\mathrm{C} . \mathrm{V} 2$ với $\mathrm{C} . \mathrm{V} 1$.

$\mathrm{m}_{\mathrm{c1}}$ - Lưu lượng nước ngưng chảy ra khỏi bộ trao đổi nhiệt. 
Ký hiệu T được tính theo độ Kelvin

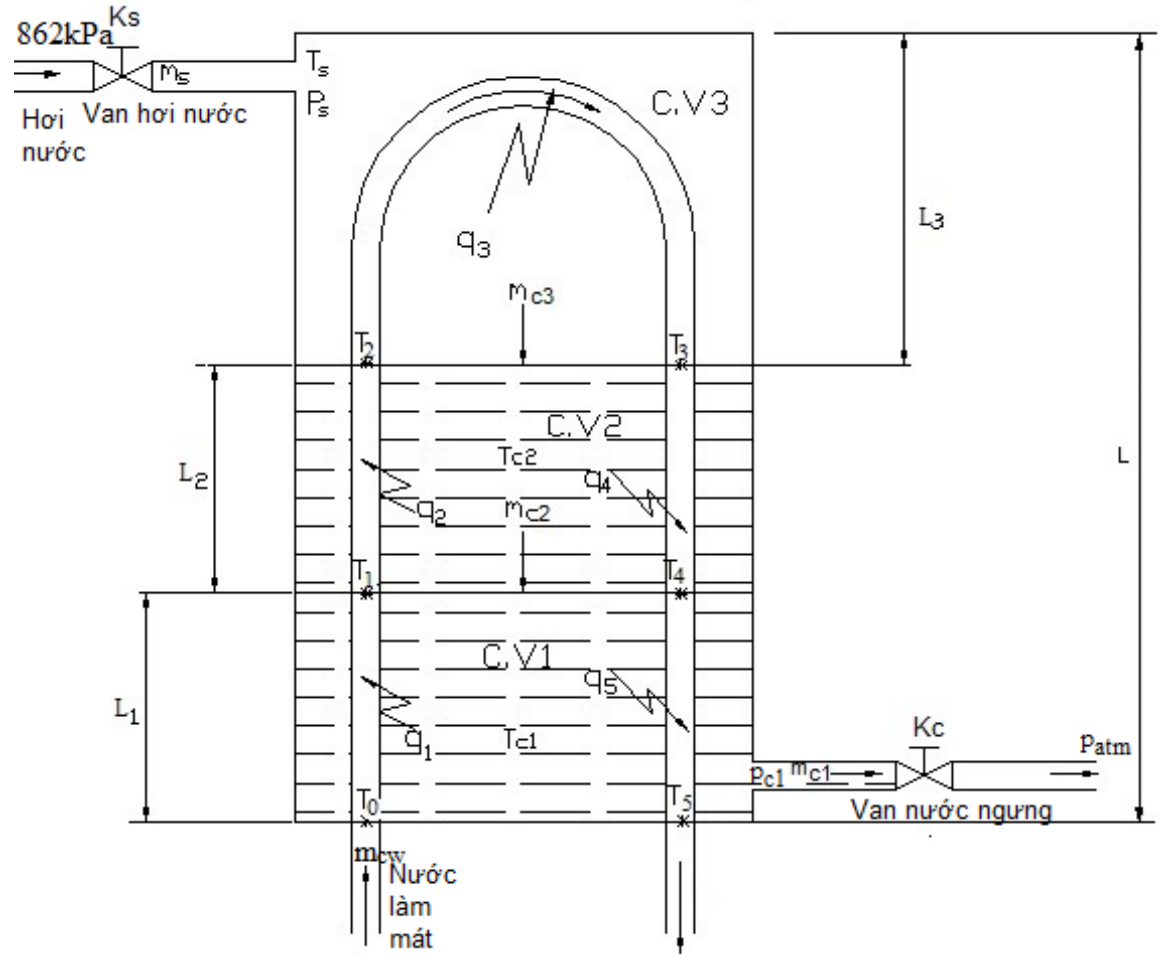

Hình 3: Mô hình hóa của bộ trao đổi nhiệt đứng kiểu ống và vỏ.

$\mathrm{L}_{1}, \mathrm{~L}_{2}, \mathrm{~L}_{3}-$ Chiều cao tương ứng của mỗi thể tích kiểm soát C.V1, C.V2 và C.V3.

$\mathrm{P}_{\mathrm{c} 1}$ - Áp suất của nước ngưng ở phía dưới.

$\mathrm{q}_{1}, \mathrm{q}_{2}, \mathrm{q}_{3}, \mathrm{q}_{4}, \mathrm{q}_{5}$ - tỉ lệ truyền nhiệt từ $\mathrm{C} . \mathrm{V}$ vỏ vào $\mathrm{C} . \mathrm{V}$ ống.

Ngoài ra, các thông số được biết là nhiệt độ $\mathrm{T}_{0}$ nước làm mát và lưu lượng nước làm mát $\mathrm{m}_{\mathrm{cw}}$, áp suất vào của hơi nước và hệ số $\mathrm{K}_{\mathrm{s}}, \mathrm{K}_{\mathrm{c}}$ của 2 van điều khiển lưu lượng của hơi nước đi vào bộ trao đổi nhiệt và nước ngưng ra khỏi bộ trao đổi nhiệt.

Chúng ta cần có 22 phương trình để giải quyết 22 ẩn số của mô hình động của bộ trao đổi nhiệt. Đầu tiên chúng ta có 3 phương trình cân bằng khối lượng cho 3 thể tích kiểm soát của vỏ. Ở đây chúng tôi trình bày một phương trình đại diện cho thể tích kiểm soát đầu tiên C.V1,

$$
\dot{\mathrm{m}}_{\mathrm{c} 2}-\dot{\mathrm{m}}_{\mathrm{c} 1}=\rho_{\mathrm{c} 1} \mathrm{~A} \frac{\mathrm{dL}}{\mathrm{dt}}
$$

mà $\mathrm{A}=\pi \frac{\mathrm{D}_{\mathrm{s}}^{2}}{4}-\mathrm{N} * \pi \frac{\mathrm{D}_{\mathrm{t}}^{2}}{4}$ là diện tích mặt cắt ngang của mặt vỏ; $\dot{\mathrm{m}}_{\mathrm{c} 3}, \dot{\mathrm{m}}_{\mathrm{c} 2}$ : lưu lượng nước ngưng ở biên $\mathrm{C} . \mathrm{V} 3$ với $\mathrm{C} . \mathrm{V} 2$ và lưu lượng ở biên $\mathrm{C} . \mathrm{V} 2$ với $\mathrm{C} . \mathrm{V} 1 ; \rho_{\mathrm{c} 1}$ là mật độ nước ngưng trong C.V1.

Ba phương trình tương tự từ nguyên lý bảo toàn năng lượng cho ba thể tích kiểm soát của vỏ. Phương trình đại diện cho cho C.V1, ta có

$$
\dot{\mathrm{m}}_{\mathrm{c} 2} \mathrm{C}_{\mathrm{p} 2} \mathrm{~T}_{\mathrm{c} 2}-\dot{\mathrm{m}}_{\mathrm{c} 1} \mathrm{C}_{\mathrm{p} 1} \mathrm{~T}_{\mathrm{c} 1}-\mathrm{q}_{1}-\mathrm{q}_{5}=\rho_{\mathrm{c} 1} \mathrm{AL}_{1} \frac{\mathrm{d}}{\mathrm{dt}}\left(\mathrm{C}_{\mathrm{p} 1} \mathrm{~T}_{\mathrm{c} 1}\right)
$$

mà $\mathrm{C}_{\mathrm{p} 1}$ là tỷ nhiệt đẳng áp. Nhiệt mất đi bởi nước làm mát trong các ống được tính từ cân bằng năng lượng trong 5 thể tích kiểm soát của ống. Các công thức sau trong [15] được dùng cho mỗi thể tích kiểm soát của ống. Đối với thể tích kiểm soát đầu tiên của ống, chúng ta có 


$$
\mathrm{q}_{1}=\dot{\mathrm{m}}_{\mathrm{cw}} \mathrm{C}_{\mathrm{p}}\left(\mathrm{T}_{1}-\mathrm{T}_{0}\right)
$$

và áp dụng phương trình truyền nhiệt "độ chênh nhiệt độ trung bình logarit" phát triển cho bộ trao đổi nhiệt kiểu ống và vỏ trong [15]

15-16)

$$
\mathrm{q}_{1}=\mathrm{UA}_{1} \frac{\mathrm{T}_{1}-\mathrm{T}_{0}}{\ln \frac{\mathrm{T}_{\mathrm{cl}}-\mathrm{T}_{0}}{\mathrm{~T}_{\mathrm{c} 1}-\mathrm{T}_{1}}}
$$

mà trong đó $\mathrm{UA}_{1}$ là hệ số truyền nhiệt tổng trong C.V1. Hệ số này phụ thuộc vào số lượng các ống $(\mathrm{N}=48)$, chiều dài ống trong mỗi thể tích kiểm soát, đường kính ống, hệ số đối lưu giữa bên ngoài và bên trong của thành ống, và độ dẫn nhiệt của vật liệu ống. Công thức cơ bản tính hệ số này được trình bày cụ

thể trong [15] và không được trình bày lại ở đây. Về phía ống, lưu lượng nước làm mát $\left(\mathrm{m}_{\mathrm{cw}}\right)$ thì không thay đổi cho tất cả các điều kiện hoạt động. Hơn nữa, nước ngưng được coi là không nén được để giả định rằng lưu lượng chảy này là không đổi trên toàn bộ thể tích ống. Chúng ta cần thêm 6 phương trình nữa để hoàn thiện mô hình hóa bộ trao đổi nhiệt. Các phương trình đó như sau:

$$
\mathrm{L}_{1}+\mathrm{L}_{2}+\mathrm{L}_{3}=\mathrm{L} \text {, }
$$

và ta cũng có [15]

$$
\mathrm{p}_{\mathrm{c} 1}=\mathrm{p}_{\mathrm{s}}+\rho_{\mathrm{c} 1}\left(\mathrm{~L}_{1}+\mathrm{L}_{2}\right) \mathrm{g}
$$

mà $\mathrm{p}_{\mathrm{c} 1}$ là áp suất của nước ngưng ở gần phía dưới đáy của bộ trao đổi nhiệt và $\mathrm{g}$ là gia tốc trọng trường $\left(\mathrm{g}=9.81 \mathrm{~m} / \mathrm{s}^{2}\right)$. Hai phương trình của van được dùng mà liên quan đến áp suất và lưu lượng [15]:

$$
\begin{aligned}
& \dot{\mathrm{m}}_{\mathrm{s}}=\mathrm{K}_{\mathrm{s}} \sqrt{\mathrm{p}_{0}-\mathrm{p}_{\mathrm{s}}} \\
& \dot{\mathrm{m}}_{\mathrm{c} 1}=\mathrm{K}_{\mathrm{c}} \sqrt{\mathrm{p}_{\mathrm{c} 1}-\mathrm{p}_{\mathrm{atm}}}
\end{aligned}
$$

Mà $\mathrm{P}_{0}$ là áp suất hơi nước bên ngoài hệ thống $\left(\mathrm{P}_{0}=862 \mathrm{kPa}\right)$ và $\mathrm{P}_{\mathrm{atm}}$ là áp suất không khí. Hai hệ số van $K_{\mathrm{s}}$ và $\mathrm{K}_{\mathrm{c}}$ được tính toán từ số liệu thực nghiệm trong [11]. Tới đây chúng ta cần thêm 2 phương trình nữa và chúng đều dựa trên những giả định. Đầu tiên là pha hơi nước được bão hòa dẫn đến mối quan hệ giữa áp suất và nhiệt độ của hơi nước. Cuối cùng chúng ta giả định chiều cao của mỗi thể tích kiểm soát C.V1 và C.V2 của pha nước ngưng có chiều dài bằng nhau:

$$
\begin{aligned}
& \mathrm{T}_{\mathrm{s}}=\mathrm{f}\left(\mathrm{p}_{\mathrm{s}}\right) \\
& \mathrm{L}_{1}=\mathrm{L}_{2}
\end{aligned}
$$

Hệ gồm 22 phương trình bao gồm một tập hợp các phương trình vi phân và đại số. Một chương trình được viết để giải quyết hệ phương trình này bằng cách sử dụng hàm 'ode15s' trong Matlab. Hàm này đã bao gồm một thuật toán để tự động điều chỉnh khoảng thời gian $\Delta \mathrm{t}$ để cho hội tụ từ điều kiện ban đầu cho đến khi đạt trạng thái ổn định. Sử dụng chương trình này cần nhập các thông số đầu vào $\left(\mathrm{K}_{\mathrm{s}}, \mathrm{K}_{\mathrm{c}}, \mathrm{T}_{1}\right.$ và

$\dot{\mathrm{m}}_{\mathrm{cw}}$ ) và các giá trị ban đầu cho 5 thông số chi phối bởi các phương trình vi phân là $\mathrm{T}_{\mathrm{cl}}, \mathrm{T}_{\mathrm{c} 2}, \mathrm{p}_{\mathrm{s}}, \mathrm{L}_{1}$ và $\mathrm{L}_{2}$.

\section{KIỂM NGHIỆM MÔ HÌNH TOÁN VÀ THẢO LUẬN KẾT QUẢ}

Hai trường hợp mô phỏng được thực hiện trong nghiên cứu này để kiểm nghiệm mô hình toán của bộ trao đổi nhiệt với các thông số đầu vào và các điều kiện ban đầu để giải hệ phương trình vi phân được trình bày trong bảng 1 . Các kết quả mô phỏng của áp suất hơi nước, $\mathrm{p}_{\mathrm{s}}$, và chiều cao mức nước ngưng tụ trong bộ trao đổi nhiệt $\mathrm{H}=\mathrm{L}_{1}+\mathrm{L}_{2}$ theo thời gian sẽ được so sánh với kết quả thực nghiệm được đo trong 
[11] và được thể hiện trên các hình 4,5 và 6 . Đường nét liền đại diện cho kết quả được đưa ra bởi mô hình toán học (mô phỏng) và đường nét đứt đại diện cho dữ liệu thực nghiệm.

Bảng 1: Các thông số đầu vào và điều kiện ban đầu cho 3 trường hợp mô phỏng.

\begin{tabular}{|c|l|l|l|}
\hline & \multicolumn{1}{|c|}{ Trường hợp 1 } & \multicolumn{1}{c|}{ Trường hợp 2 } & \multicolumn{1}{c|}{ Trường hợp 3 } \\
\hline Thông số & $-\mathrm{K}_{\mathrm{s}}=0.012 ;$ & $-\mathrm{K}_{\mathrm{s}}=0.0175 ;$ & $-\mathrm{K}_{\mathrm{s}}=0.0115 ;$ \\
đầu vào & $-\mathrm{K}_{\mathrm{c}}=0.01 ;$ & $-\mathrm{K}_{\mathrm{c}}=0.0075 ;$ & $-\mathrm{K}_{\mathrm{c}}=0.0072 ;$ \\
& $-\mathrm{T}_{1}=325 \mathrm{~K} ;$ & $-\mathrm{T}_{1}=325 \mathrm{~K} ;$ & $-\mathrm{T}_{1}=325 \mathrm{~K} ;$ \\
& $-\dot{\mathrm{m}}_{\mathrm{cw}}=0.65(\mathrm{~kg} / \mathrm{s})$ & $-\dot{\mathrm{m}}_{\mathrm{cw}}=0.65(\mathrm{~kg} / \mathrm{s})$ & $-\dot{\mathrm{m}}_{\mathrm{cw}}=0.65(\mathrm{~kg} / \mathrm{s})$ \\
\hline
\end{tabular}

Từ các hình 4,5 và 6 cho thấy sự tương đồng giữa các kết quả mô phỏng và dữ liệu thực nghiệm trong [11]. Sai số trung bình giữa kết quả mô phỏng và thực nghiệm cho áp suất hơi nước lần lượt là $0.48 \%, 0.95 \%$ và $3.98 \%$ cho các trường hợp 1,2 và 3 tương ứng. Sai số trung bình giữa kết quả mô phỏng và thực nghiệm cho mực nước ngưng lần lượt là $2.60 \%, 0.45 \%$ và $0.50 \%$ cho các trường hợp 1,2 và 3 tương ứng. Như vậy xét chung cho 3 trường hợp chiều cao của mực nước ngưng được dự đoán tốt hơn so với áp suất hơi nước. Nó có thể được giải thích bởi thực tế rằng nước ngưng là gần như không nén được và có nhiều quán tính chống lại sự thay đổi, trong khi hơi nhạy hơn với những thay đổi bên ngoài như các tác động mở van. Sai số giửa kết quả mô phỏng chiều cao mực nước ngưng có thể đến từ giả định $\mathrm{L}_{1}=\mathrm{L}_{2}$ mà ảnh hưởng đến các đại lượng như lưu lượng nước ngưng, nhiệt độ trung bình nước ngưng v.v... trong các phương trình vi phân bảo toàn khối lượng và năng lượng. Tuy nhiên sai số của giả định trên có thể được giảm nếu chúng ta tăng số thể tích kiểm soát trong thể tích nước ngưng. Các thông số trong mô hình như các hệ số van $\mathrm{K}_{\mathrm{s}}$ và $\mathrm{K}_{\mathrm{c}}$ các đặc tính van, và các hệ số truyền nhiệt cần nhiều xác định và kết quả thử nghiệm là cần thiết để đánh giá những giá trị này để đạt kết quả mô phỏng tốt hơn. Cho đến hiện tại mô hình toán học ở trên có thể dùng để mô phỏng các hiện tượng vật lý của bộ trao đổi nhiệt thẳng đứng kiểu vỏ và ống.
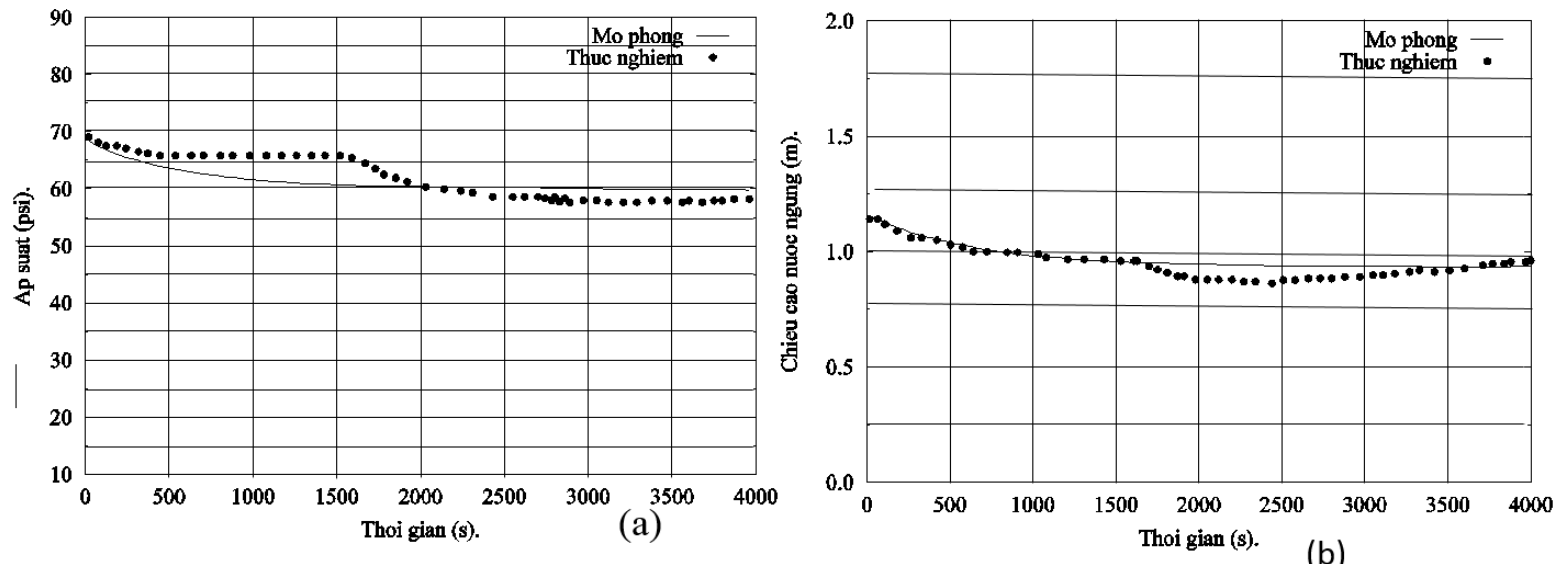

Hình 4. Các kết quả của trường hợp 1: a) Áp suất hơi nước và b) Chiều cao mực nước ngưng. 

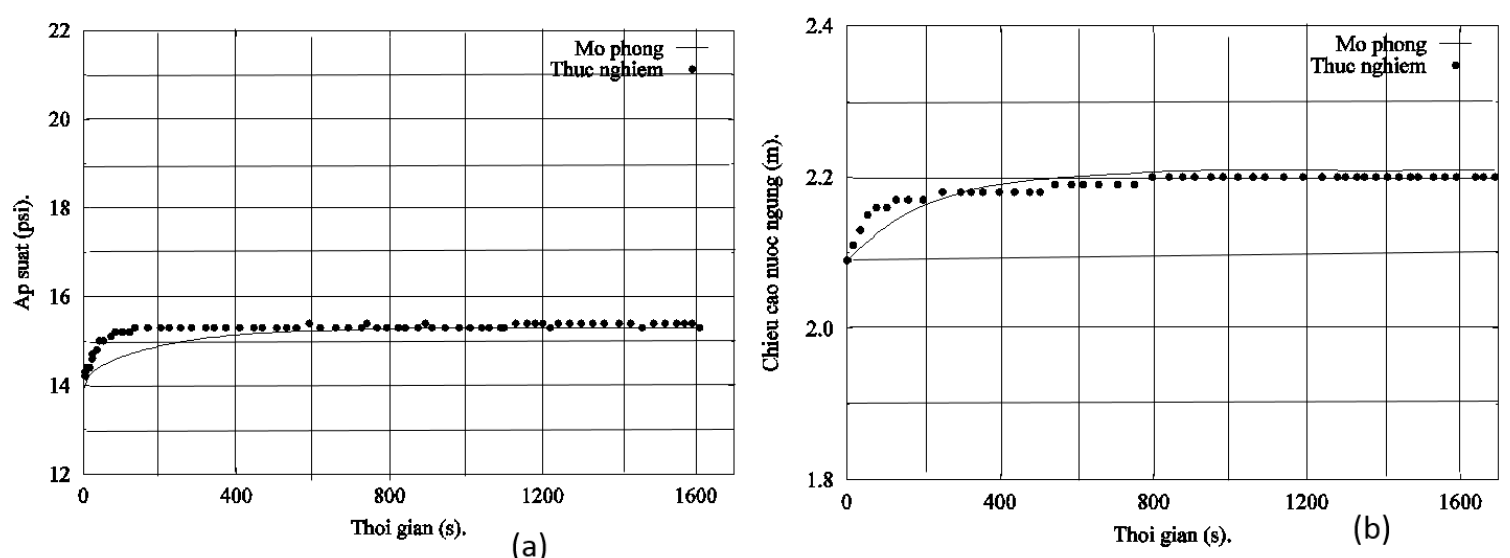

Hình 5. Các kết quả của trường hợp 2: a) Áp suất hơi nước và b) Chiều cao mực nước ngưng.
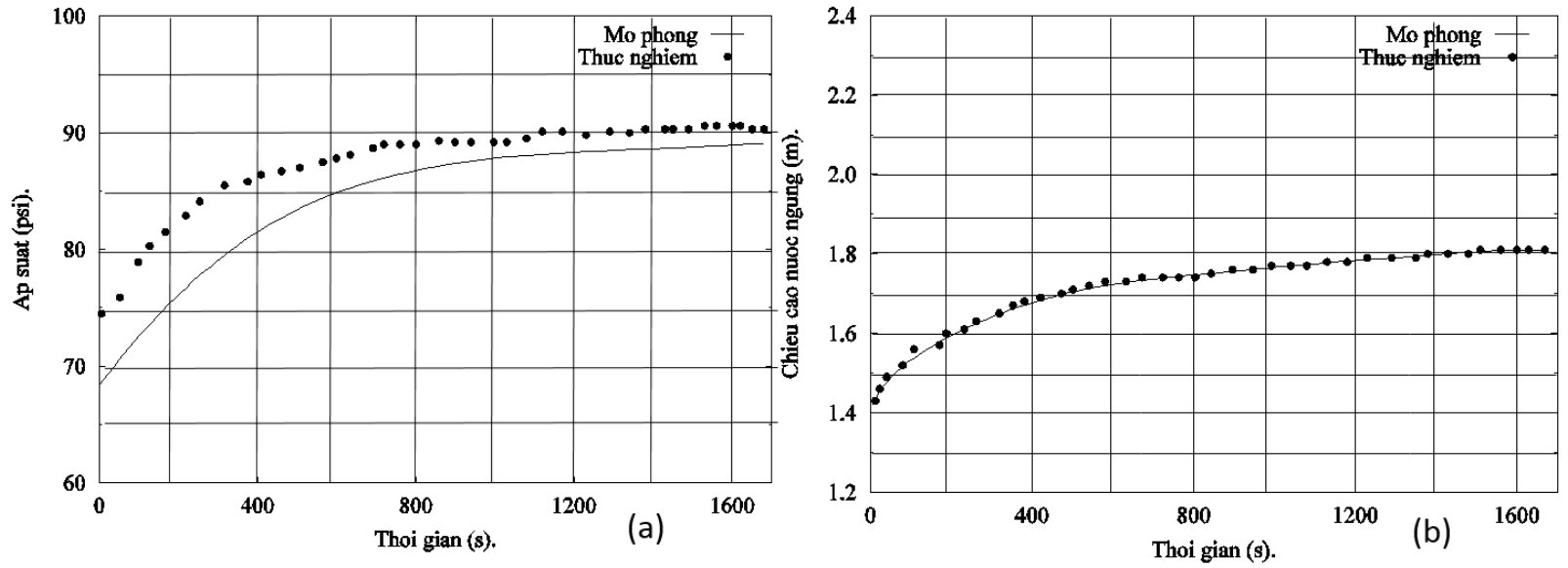

Hình 6. Các kết quả của trường hợp 3: a) Áp suất hơi nước và b) Chiều cao mực nước ngưng.

\section{KẾT LUÂN}

Bộ trao đổi nhiệt kiểu vỏ và ống đứng đã được mô phỏng thành công qua áp dụng các nguyên lý bảo toàn khối lượng và năng lượng cho các thể tích kiểm soát. Một mô hình ban đầu với 3 thể tích kiểm soát trên mặt vỏ và 5 thể tích kiểm soát tương ứng trên mặt ống dẩn đến một hệ gồm 22 phương trình vi phân và đại số đã dự đoán tốt áp suất hơi nước và mức nước ngưng trong bộ trao đổi nhiệt so với dữ liệu thực nghiệm. Mô hình toán học này trình bày một lợi thế lớn đầu tiên là khả năng mô tả các đáp ứng động tại các vị trí khác nhau của bộ trao đổi nhiệt, và thứ hai là mối quan hệ có ý nghĩa giữa các tham số. Những mối quan hệ cho phép đánh giá về tính hợp lý của các đáp ứng. Những dự đoán từ mô hình toán có thể có giá trị trong việc áp dụng các mô phỏng cho các mục đích cụ thể như tối ưu hóa hệ thống điều khiển của hệ thống đo lưu lượng.

\section{TÀI LIỆU THAM KHẢO}

[1]. White, J.R., Lecture notes, System Dynamics, Uni of Mass-Lowell, 1997.

[2]. Botch T.W. Alcock S.K., Webb D.R., Modelling and simulating of the dynamic behaviour of a Shell and Tube Condenser. Int, J. Heat ctnd Mass Transfer, 1997, 40(17) : 4137-4149.

[3]. Vera-García F., García-Cascales J.R. , Gonzálvez-Maciá J., Cabello R., Llopis R., Sanchez D. Torrella E., A simplified model for shell-and-tubes heat exchangers: Practical application, Applied Thermal Engineering, 2010, 30(10):1231-1241. 
[4]. A. Sodja, B. Zupancic, J. Sink, Some aspects of the modeling of tube-and-shell heat-exchangers, Proceedings 7th Modelica Conference, Como, Italy, $2009: 716$ - 721.

[5]. Daniel J. C, Jacinto L. M., Dynamic Simulation of Shell-and-Tube Heat Exchangers, Heat Transfer Engineering, 1987, 8: 50-59.

[6] D. Singh, J. Pandey and A. Tiwari, CFD analysis of a shell and tube heat exchanger using different header sections, International journal of research in aeronautical and mechanical engineering, 2016, 4: 1- 10.

[7] X. Gu, Q. Dong, and K. Wang, Numerical simulation research on shell and tube heat exchanger based on 3D solid model, Proceedings of the International conference on power engineering and enviroment, 2007, 441445 .

[8] M. Vukic, G. Vuckovic, P. Zivkovic, Z. Stevanovic and M. Tomic, 3D numerical simulations of the thermal processes in the shell and tube heat exchanger, Facta universitatis, series: Mechanical Engineering, 2013, 11:169-180.

[9] N. V. Raja, B. Jithendra, Modeling and analysis of shell and tube heat exchanger tubes by using finite element analysis, International Journal and Magazine of Engineering Technology, Management and research, 2015, 2: 910-914.

[10] J. Zhang, Y. He, W. Tao, 3D numerical simulation on shell-and-tube heat exchangers with middle-overlapped helical baffles and continuous baffles - Part I: Numerical model and results of whole heat exchanger with middle-overlapped helical baffles, International Journal of Heat and Mass Transfer, 2009, 52: 5371-5380.

[11]. Vincent N., Développement d'une méthode de calibration des débitmètres de vapeur basée sur la mesure du poids du condensât. Mémoire de maitrise, École de Technologie Supérieure, 2002.

[12]. http://ctt.etsmtl.ca, 08/2016.

[13]. Kajl, S., Lamarche, L., Masson,C., Développement d'une méthode de calibration des débitmètres de vapeur basée sur la mesure du poids de condensât : V Colloque interuniversitaire franco - québécois, Lyon, 2001.

[14]. Kaj1, S., Masson, C., Lamarche, L 2000.: Centre for thermal technology activities, International Conference Problems of environment engineering at the threshold of the new millennium, Wroclaw - Szklarska Poreba, Pologne, 2000: 301- 306.

[15]. Incropera F.P., DeWitt D.P., Introduction to Heat and Mass Transfer, Fourth Edition, John Wiley and Son, 1996.

Ngày nhận bài: 28/01/2017

Ngày chấp nhận đăng: 10/09/2017 